\title{
M2000, an Antagonist for Human TLR2 and TLR4
}

\author{
Mona Aslani ${ }^{1}$ and Abbas Mirshafiey*1,2 \\ ${ }^{1}$ Department of Immunology, School of Public Health, Tehran University of Medical Sciences, Tehran, Iran. \\ ${ }^{2}$ Research Centre for Immunodeficiencies, Tehran University of Medical Sciences, Tehran, Iran
}

*Corresponding author: Abbas Mirshafiey, Department of Immunology, School of Public Health, Tehran University of Medical Sciences, Tehran, Iran.

Received Date: December 16, 2018

Published Date: May 23, 2019

\begin{abstract}
Toll-like receptors (TLRs) especially TLR2 and TLR4 play a crucial role in immunopathogenesis of autoimmune and inflammatory diseases such as rheumatoid arthritis (RA) and ankylosing spondylitis (AS); therefore, selective blockade of these receptors or associated adaptor molecules in their signaling pathways has been developed, as a new therapeutic approach for many inflammatory diseases. The $\beta$-D-Mannuronic acid (M2000) is a new anti-inflammatory drug with immunosuppressive properties. Several in vitro and in vivo investigations have shown that this new drug is able to target the TLR2 and TLR4 signaling pathways and act as an antagonist for these receptors.
\end{abstract}

Keywords: M2000; Mannuronic acid; TLR2; TLR4; NSAIDs

\section{Introduction}

TLRs which are single, type I transmembrane glycoproteins are expressed by different types of immune cells such as monocytes/ macrophages, dendritic cells and neutrophils. They identify invading organisms including Pathogen Associated Molecular Patterns (PAMPs) and Damage Associated Molecular Patterns (DAMPs) [12]. There are 13-15 TLR types in mammals and among them, TLR113 have been identified in humans and mice[3]. Structurally, TLRs have several domains, including ectodomains with leucine rich repeat (LRR) motifs which expands into the extracellular region, some transmembrane domains; intracellular Toll-interleukin 1 (IL-1) receptor (TIR) domains and a Toll/interleukin-1 receptor (IL-1R) - interacting (TIR) domain which is vital for intracellular signal transduction. Ligation of TLRs provokes two distinct pathways with different and sometimes overlapping results: the Myeloid Differentiation primary response protein 88 (MyD88)dependent and the TIR-domain-containing adapter inducing interferon- $\beta$ (TRIF)-dependent pathways [2,4]. MyD88 interacts with TIR domain of TLRs and then with IL-1R-associated kinase 1 and 4 (IRAK1,4), which leads to activation of NF-kB and AP-1 transcription factors along with the up-regulation of inflammatory cytokines and mediators gene [4]. These receptors regulate a pro-/anti-inflammatory balance. Between the TLRs, TLR2 is able to detect a variety of microbial products, including lipoproteins, lipoteichoic acid (LTA) and peptidoglycan, by formation of heterodimers with other kinds of TLRs [1,3]. Activation of TLR2 results in stimulation of both MyD88-dependent and independent pathways, which can regulate the balance between cell survival and inflammation [3]. TLR4 is also the principal recognition receptor of Lipopolysaccharide (LPS), and can be activated by both MyD88dependent and independent pathways [4]. TLRs, especially TLR2 and TLR4 have a key role in autoimmune and inflammatory diseases, and single nucleotide polymorphisms in their genes have a close relationship with some disorders [3,4]. They are associated with a variety of diseases, such as RA, AS, inflammatory bowel disease (IBD), multiple sclerosis (MS) and systemic lupus erythematosus (SLE); therefore, they are considered as therapeutic targets and the use of their antagonists as well as their signal transduction inhibitors have been proposed as desirable therapeutic strategies for some autoimmune and inflammatory disorders [2-4].

The $\beta$-D-Mannuronic acid (M2000) (C6H10O7) patented (DE; 102016113018.4), is a new anti-inflammatory drug belongs to the Non-Steroidal Anti-Inflammatory Drugs (NSAIDs) family with a very low molecular weight (194.139 Da), together with immunosuppressive properties, which has been demonstrated in the numerous studies. This monomer is generated directly from Alginic acid, which there is abundantly in the cell walls of brown algae and is used in the cosmetics and food industries. The preliminary researches showed its potential therapeutic effects 
in numerous animal models consist of Adjuvant-Induced Arthritis (AIA), experimental autoimmune encephalomyelitis (EAE), glomerulonephritis and nephrotic syndrome. In addition, in some other investigations, its remarkable tolerability and biocompatibility have been proved, which are accompanied by the lack of cytotoxic effects in comparison to the other members of NSAIDs family, such as Diclofenac and Piroxicam and even Corticosteroids, such as dexamethasone [1-34]. A considerable point about this drug, which has been proved in some clinical trial investigations, is that unlike the other kinds of NSAIDs, the oral administration of M2000 not only hasn't any to low side effects, but also it can even decline the side effects of another simultaneous consumed drugs in patients [23]. Recently a phase I/II randomized, controlled, clinical trial for evaluating the effects and safety of M2000 in RA patients, has been done. In this trial, the M2000-treated patients showed a significant improvement, and a great significant reduction in their disease activity score 28 (DAS28) index was observed [23]. Based on the molecular structure of M2000, its cell surface receptors could be probably the members of mannose receptors (MR) family, while, TLR2 and TLR4 can be the second group of receptors of this drug [1].

\section{Effects of M2000 on TLR2 and TLR4 Under In Vitro Investigations}

Aletaha S et al. [4], using human embryonic kidney cell line (HEK293-Blue TLR-2/-4), a highly useful cell line for evaluating the TLRs signaling, have shown that in HEK-Blue hTLR2 cells, the mRNA expression of MyD88 and NF-kB were reduced significantly, after treatment of the cells with low and high concentrations of M2000 ( 5 and $25 \mu \mathrm{g}$ /well, respectively) alone or in combination with LTA, in comparison to unstimulated control and LTA treated cells; while, in HEK-Blue hTLR4 cells, M2000 (both at 5 and $25 \mu \mathrm{g} /$ well) in combination with LPS decreased the mRNA expression of MyD88 in comparison to LPS, and the mRNA expression of NF-kB declined in these cells after treatment with both 5 and $25 \mu \mathrm{g} /$ well of M2000 in combination with LPS, in comparison to unstimulated control cells and LPS, respectively. They have also reported that in both HEKBlue hTLR2 and HEK-Blue hTLR4 cells, TNF- $\alpha$ and IL- 6 production reduced significantly, after treatment of the cells with both low and high concentrations of M2000 in combination with LTA and LPS, respectively [4]. Furthermore, Mortazavi-Jahromi SS, et al [3] using HEK-Blue hTLR2 cell line have shown that after treatment of the cells with both low and high concentrations of M2000 (5 and $25 \mathrm{ug}$ / well, respectively), the mRNA expression of IRAK1, TNF receptorassociated factor 6 (TRAF6), miR-146a and nuclear factor-kB (NF$\mathrm{kB}$ ) reduced significantly, in comparison to control group. It should be noted that, miR-146a play a key role in innate immunity and its expression is associated with NF-kB gene activity. It is involved in immunopathogenesis of several autoimmune diseases such as RA, SLE and Sjögren's syndrome (SS); therefore, down-regulation of this molecule and its target mediators can lead to controlling the inflammation [3]. Moreover, Sharifi L et al; 2018, using HT29 cell line (a colonic epithelial cell model) have reported that both low and high doses of M2000 (5 and $25 \mu \mathrm{g} /$ well) significantly down- regulated the mRNA expression of both TLR2 and TLR4; in addition, treatment of the cells with high dose of M2000 in combination with LPS, reduced the mRNA expression of TLR2 and TLR4, significantly [35]. Pourgholi F et al. [1] by utilizing HEK293-TLR2 cell line have also shown that Src Homology-2 domain-containing inositol-5'phosphatase 1 (SHIP1) and Suppressors of Cytokine Signaling 1 (SOCS1) increased significantly, after treatment the cells with both 5 and $25 \mu \mathrm{g} / \mathrm{ml}$ of M2000. The SOCS family components play a key role in a negative feedback loop that regulates the intensity and duration of cytokine signaling. SOCS1 controls LPS induced inflammation negatively, thus, inhibits the secretion of pro-inflammatory cytokines such as, TNF- $\alpha$, IL- 6 and INF- $\gamma$. SHIP1 is other target in TLR's signaling pathways, which regulates the activation of immune cells negatively, mostly through the phosphoinositide 3-kinase (PI3K) pathway; therefore, down-regulation of these mediators leads to the reduction of inflammation. Furthermore, they have reported that treatment of the Peripheral Blood Mononuclear Cells (PBMCs) of healthy individuals with both low and high doses of M2000 in combination with LPS significantly down-regulated the mRNA expression of miR-155 compared to LPS treated cells. As miR-155 is a substantial regulator of innate immunity and TLR signaling pathway through the targeting SOCS1 and SHIP1 effects in inflammatory responses, therefore, declining this molecule can results in controlling the inflammation[1].

\section{Effects of M2000 on TLR2 and TLR4 Under In Vivo Investigations}

Roozbehkia M, et al. [2] using PBMCs of patients suffering from active form of AS, have shown that following the oral administration of $1000 \mathrm{mg} /$ day of M2000 for 12 weeks by the patients, the mRNA expression of IKB-alpha, Myd88 and NF-kB reduced significantly in comparison to before treatment with M2000. Many studies have shown that TLRs have a potential role in the pathogenesis of AS and $\beta$-D-mannuronic acid reduced production of inflammatory mediators in these patients, through interfering the TLR/NF-kB Signaling Pathway [2].

Collectively, regarding the key role of the TLRs in the autoimmune and inflammatory diseases, using their antagonists can be considered as an important therapeutic target, and based on the results of the above mentioned investigations, it seems that M2000, as a monomer molecule of sodium alginate, can probably act as an antagonist for TLR2 and TLR4, and consequently, reduce the inflammation.

\section{Acknowledgement}

None.

\section{Conflict of Interest}

No Conflict of Interest.

\section{References}

1. Pourgholi F, Hajivalili M, RazaviR, Esmaeili S, Baradaran B, et al. (2017) The role of M2000 as an anti-inflammatory agent in toll-like receptor 2/ microRNA-155 pathway. Avicenna J Med Biotechnol 9(1): 8-12.

2. Roozbehkia M, Mahmoudi M, Aletaha S, Rezaei N, Fattahi MJ, et al. (2017) The potent suppressive effect of $\beta$-d-mannuronic acid (M2000) on 
molecular expression of the TLR/NF-kB Signaling Pathway in ankylosing spondylitis patients, Int Immunopharmacol 52: 191-196.

3. Mortazavi-Jahromi SS, Jamshidi MM, Farazmand A, Aghazadeh Z, Yousefi, et al. (2017) Pharmacological effects of $\beta$-d-mannuronic acid (M2000) on miR-146a, IRAK1, TRAF6 and NF- $\kappa$ B gene expression, as target molecules in inflammatory reactions. Pharmacological reports 69(3): 479-484.

4. Aletaha S, Haddad L, Roozbehkia M,Bigdeli R, Asgary V, et al. (2017) M2000 ( $\beta$-D-Mannuronic Acid) as a Novel Antagonist for Blocking the TLR 2 and TLR 4 Downstream Signalling Pathway.Scandinavian journal of immunology 85(2): 122-129.

5. Mirshafiey A, Cuzzocrea S, Rehm B,Mazzon E, Saadat F, et al. (2005) Treatment of experimental arthritis with M2000, a novel designed nonsteroidal anti-inflammatory drug.Scandinavian journal of immunology 61(5): 435-441.

6. Mirshafiey A, Abbas, Cuzzocrea S, Rehm B, Matsuo H,et al.(2005)M2000: a revolution in pharmacology, Medical science monitor, 11(8): PI53PI63.

7. Mirshafiey A,Khorramizadeh MR, Saadat F, Rehm BH (2004) Chemopreventive effect of M2000, a new anti-inflammatory agent, Medical Science Monitor 10: PI105-PI109.

8. Mirshafiey A, Matsuo H, Nakane S, Rehm BH, Koh CS, et al. (2005) Novel immunosuppressive therapy by M2000 in experimental multiple sclerosis, Immunopharmacology and immunotoxicology 27: 255-265.

9. Mirshafiey A, Rehm B, Abhari RS, Borzooy Z, Sotoude M, et al. (2007) Production of M2000 ( $\beta$-d-mannuronic acid) and its therapeutic effect on experimental nephritis, Environmental toxicology and pharmacology 24(1): 60-66.

10. Mirshafiey A,Rehm B, Sotoude M, Razavi A, Abhari RS, et al. (2007) Therapeutic approach by a novel designed anti-inflammatory drug, M2000, in experimental immune complex glomerulonephritis. Immunopharmacology and immunotoxicology 29(1): 49-61.

11. Mirshafiey A, Rehm BH, Sahmani AA, Naji A, Razavi A (2004) M-2000, as a new anti-inflammatory molecule in treatment of experimental nephrosis, Immunopharmacology and immunotoxicology 26: 611-619.

12. Fattahi MJ, Abdollahi M, Agha Mohammadi A, Rastkari N, KhorasaniR, et al. (2015) Preclinical assessment of $\beta$-d-mannuronic acid (M2000) as a non-steroidal anti-inflammatory drug, Immunopharmacology and immunotoxicology 37: 535-540.

13. Farahani MM, Motevaseli E, Maghsood F, Heidari-KharajiM,Mirshafiey $A(2017)$ Anti-inflammatory property of $\beta$-D-mannuronic acid (M2000) on expression and activity of matrix metalloproteinase- 2 and-9 through CD147 molecule in phorbol myristate acetate-differentiated THP-1 cells. Iranian journal of allergy, asthma and immunology 16(5): 443-451.

14. Barati A, Jamshidi AR, Ahmadi H, Aghazadeh Z, Mirshafiey A,(2017) Effects of $\beta$-D-mannuronic acid, as a novel non-steroidal antiinflammatory medication within immunosuppressive properties, on IL17, ROR $\gamma$ t, IL4 and GATA3 gene expressions in rheumatoid arthritis patients, Drug design, development and therapy 11: 1027-1033

15. Ahmadi H, Mahmoudi M, Gharibdoost F, Vojdanian M, Areza Jamshidi A (2018) Targeting of circulating Th17 cells by $\beta$-D-mannuronic acid (M2000) as a novel medication in patients with rheumatoid arthritis, Inflammopharmacology 26: 57-65.

16. Ahmadi H, Jamshidi AR, Mahmoudi M, Gharibdoost F, Vojdanian M, et al. (2017)Hematological improvement of patients with active rheumatoid arthritis by $\beta$-d-mannuronic acid (M2000) as a novel NSAID with immunosuppressive property. Iranian Journal of Allergy, Asthma and Immunology 16(5): 433-442.

17. Jafarnezhad-Ansariha F, Yekaninejad MS,Jamshidi AR, Mansouri R, Vojdanian M,et al. (2018)The effects of $\beta$-d-mannuronic acid (M2000), as a novel NSAID, on COX1 and COX2 activities and gene expression in ankylosing spondylitis patients and the murine monocyte/macrophage, J774 cell line. Inflammopharmacology 26(2): 375-384.

18. Fattahi MJ, Ahmadi H, Jafarnezhad-Ansariha F, Mortazavi-Jahromi SS, Rehm BH, et al. (2018) Oral administration effects of $\beta$-d-mannuronic acid (M2000) on Th17 and regulatory T cells in patients with ankylosing spondylitis. Biomedicine \& Pharmacotherapy 100: 495-500.

19. Mohammed HA, Saboor-Yaraghi AA, Vahedi H, Yekaninejad MS, Panahi $G$, et al. (2017) immunomodulatory effects of M2000 ( $\beta$-d-mannuronic acid) on Tnf- $\alpha$, Il-17 and Foxp3 gene expression in patients with inflammatory bowel disease, International immunopharmacology, 51 :107-113.

20. Tabrizian N, Arjomand Fard N, Farazmand A, Eftekhari R,Tofighi Zavareh F, et al. (2017)The Biology of $\beta$-D-mannuronic acid (M2000) on Human Dendritic Cell Based on MicroRNA-155 and MicroRNA-221, Current drug discovery technologies, 14 (1):53-58.

21. Hosseini S, Abdollahi M, Azizi G, Fattahi MJ, Rastkari N, et al.(2017) Anti-aging effects of M2000 ( $\beta$-D-mannuronic acid) as a novel immunosuppressive drug on the enzymatic and non-enzymatic oxidative stress parameters in an experimental model, Journal of basic and clinical physiology and pharmacology, 28 (3):249-255.

22. Fard NA, Tabrizian N, Mirzaei R, Motamed N,Zavareh FT, et al. (2016) The Safety Property of $\beta$-D-Mannuronic Acid (M2000) as a Novel Immunosuppressive Agent on Differentiation, Maturation and Function of Human Dendritic Cells, Current drug discovery technologies, 13 (3): 164-169.

23. Ahmadi H,Jamshidi AR, Gharibdoost F, Mahmoudi M, Rastkari N, et al.(2018)A phase I/II randomized, controlled, clinical trial for assessment of the efficacy and safety of $\beta$-d-mannuronic acid in rheumatoid arthritis patients, Inflammopharmacology 26 (3):737-745.

24. Ahmadi H, Jamshidi AR, Mahmoudi M, Cuzzocrea S, Fattahi MJ, et al.(2017)The potent inhibitory effect of $\beta$-D-mannuronic acid (M2000) as a novel NSAID with immunosuppressive property on anti-cyclic citrullinated peptide antibodies, rheumatoid factor and anti-dsDNA antibodies in patients with rheumatoid arthritis, Current drug discovery technologies, 14 (3): 206-214.

25. Athari Nik Azm S, Vafa M, Sharifzadeh M,Safa M, et al.(2017) Effects of M2000 (D-mannuronic acid) on learning, memory retrieval, and associated determinants in a rat model of Alzheimer's disease, American Journal of Alzheimer's Disease \& Other Dementias® 32 (1): 12-21.

26. Fattahi MJ, Jamshidi AR, Mahmoudi M,Vojdanian M,Yekaninejad MS, et al.(2018) Evaluation of the efficacy and safety of $\beta$-d-mannuronic acid in patients with ankylosing spondylitis: a 12-week randomized, placebocontrolled, phase I/II clinical trial, International immunopharmacology $54: 112-117$.

27. Hosseini F, Hassannia H,Mahdian-Shakib A, Jadidi-Niaragh F, Enderami SE,et al.(2017)Targeting of crosstalk between tumor and tumor microenvironment by $\beta$-D mannuronic acid (M2000) in murine breast cancer model, Cancer medicine 6 (3): 640-650.

28. Jahanbakhshi M, Babaloo Z, Mortazavi-Jahromi SS,Shokri MM, et al.(2018) Modification of Sexual Hormones in Rheumatoid Arthritis Patients by M2000 ( $\beta$-D-mannuronic Acid) as a Novel NSAID with Immunosuppressive Property, Endocrine, Metabolic \& Immune Disorders-Drug Targets (Formerly Current Drug Targets-Immune, Endocrine \& Metabolic Disorders) 18 (5): 530-536.

29. Mirshafiey A, Taeb M, Mortazavi-Jahromi SS, Jafarnezhad-Ansariha F, Rehm BH, et al.(2017) Introduction of $\beta$-d-mannuronic acid (M2000) as a novel NSAID with immunosuppressive property based on COX-1/COX2 activity and gene expression, Pharmacological reports 69 (5) :10671072

30. Mortazavi-Jahromi SS,Alizadeh S, Javanbakht MH, Mirshafiey A (2018) Anti-diabetic effect of $\beta$-D-mannuronic acid (M2000) as a novel NSAID with immunosuppressive property on insulin production, blood glucose, and inflammatory markers in the experimental diabetes model, Archives of physiology and biochemistry p.1-6.

31. Mortazavi-Jahromi SS, Alizadeh S,Javanbakht MH, Mirshafiey A(2018) Cardioprotective effect of $\beta$-d-mannuronic acid (M2000) as a novel NSAID on gene expression of oxLDL scavenger receptors in the experimental diabetic model, Immunopharmacology and immunotoxicology 40(4):284-289.

32. Rastegari-Pouyani M, Mostafaie A,Mansouri K, S Mortazavi-Jahromi SS,Mohammadi-Motlagh HR, et al.(2018)Anti-angiogenesis effect of $\beta$-D- 
mannuronic acid (M2000) as a novel NSAID with immunosuppressive properties under experimental model, Clinical and Experimental Pharmacology and Physiology 45 (4): 370-376.

33. Taeb M, Jafarzadeh A, Mortazavi-Jahromi SS, Zainodini N, Mirzaei M, et al.(2018) Effect of $\beta$-D-mannuronic acid (M2000) on oxidative stress enzymes' gene using healthy donor peripheral blood mononuclear cells for evaluating the anti-aging property, Current drug discovery technologies.
34. Vojdanian M, Ahmadi H, Reza Jamshidi A, Mahmoudi M,Gharibdoost F, et al. (2017) The Anti-Migraine Effects of M2000 ( $\beta$-D-Mannuronic Acid) on a Patient with Rheumatoid Arthritis: Case Report, Current clinical pharmacology, 12 (2): 127-130.

35. Sharifi L MM, Dallal MMS, Asgardoon MH, Nourizadeh M, Bokaie S, et al. (2018)The Inhibitory Role of M2000 ( $\beta$-D-Mannuronic Acid) on Expression of Toll-like Receptor 2 and 4 in HT29 Cell Line., Inflammation and allergy drug discovery. 